\title{
Energy Efficient Video Compression for Wireless Sensor Networks
}

\author{
Junaid Jameel Ahmad ${ }^{1,2}$, Hassan Aqeel Khan ${ }^{2}$, and Syed Ali Khayam ${ }^{2}$ \\ ${ }^{1}$ College of Signals, ${ }^{2}$ School of Electrical Engineering \& Computer Science \\ National University of Sciences and Technology (NUST), \\ Sector H-12, Islamabad, Pakistan. \\ junaidjahmad@mcs.edu.pk, \{hassan.aqeel, khayam\}@niit.edu.pk
}

\begin{abstract}
Wireless video sensor networks are anticipated to be deployed to monitor remote geographical areas. To save energy in bit transmissions/receptions over a video sensor network, the captured video content needs to be encoded before its transmission to the base station. However, video encoding is an inherently complex operation that can cause a major energy drain at battery-constrained sensors. Thus a systematic evaluation of different video encoding options is required to allow a designer to choose the most energy-efficient compression technique for a given video sensing application scenario. In this paper, we empirically evaluate the energy efficiencies of predictive and distributed video coding paradigms for deployment on real-life sensor motes. For predictive video coding, our results show that despite its higher compression efficiency, inter video coding always depletes much more energy than intra coding. Therefore, we propose to use image compression based intra coding to improve energy efficiency in the predictive video coding paradigm. For distributed video coding, our results show that the Wyner-Ziv encoder has consistently better energy efficiency than the PRISM encoder. We propose minor modifications to PRISM and Wyner-Ziv encoders which significantly reduce the energy consumption of these encoders. For all the video encoding configurations evaluated in this paper, our results reveal the counter-intuitive and important finding that the major source of energy drain in WSNs is local computations performed for video compression and not video transmission.
\end{abstract}

\section{INTRODUCTION}

Due to their ease of deployment and dynamically configurable nature, wireless video sensor networks are now becoming increasingly popular [1], [2]. In particular, video sensor networks are finding applications in remote area monitoring and surveillance, such as monitoring of battlefields, disasterstruck regions, forests, and wildlife habitats [2]. Wireless sensor networks (WSNs) are particularly well-suited for such (mostly hostile) deployment scenarios because of their selfconfiguring and self-organizing characteristics. However, the constraints of WSNs directly conflict with the requirements of video communication. In particular, due to limited energy resources at sensor motes, the most fundamental constraint on any WSN application or protocol is energy efficiency.

Two main sources of energy drain in a sensor mote are local computations and bit transmissions/receptions. Video communication incurs both types of energy depletion as video

\footnotetext{
${ }^{*}$ This work is supported by Nokia Research, China.
}

content is bandwidth-intensive (requiring significant bit transmissions/receptions) and video compression is an inherently complex operation (requiring significant local computations). In this context, video communication over WSNs presents an interesting tradeoff. On the one hand, in video sensors the idea of performing a high degree of compression to reduce the number of bit transmissions/receptions seems appealing. On the other hand, use of a complex compression technique will result in a considerable increase in the number of local computations at a sensor. A systematic comparison/investigation of the energy depletion of different video coding alternatives on actual wireless video sensors is required to study this tradeoff.

In this paper, we present experimental results of energy consumption due to video compression and transmission for predictive and distributed ${ }^{1}$ video encoding paradigms over the Stargate [3], [4] sensor platform. Moreover, we propose simple modifications to existing video encoders to improve their energy efficiency. For the Predictive Video Coding (PVC) paradigm, we first evaluate the energy consumption for inter- and intra-coded H.264 [5]-[7] video compression. Our experimental results indicate that inter-coded video always consumes much higher amounts of energy (on average $763.68 \mathrm{~mJ} /$ frame) in comparison to intra-coded video $(60.03 \mathrm{~mJ} /$ frame $)$. Since intra-coding provides better energy efficiency than motion compensated video coding, for the PVC case we propose to use a simple image coding system instead of a conventional video coder. In this context, we show that Motion JPEG2000 [8], [9] can provide much better energy efficiency $(15.15 \mathrm{~mJ} /$ frame) than H.264 intra coding.

Under the Distributed Video Coding (DVC) paradigm [10][16], we evaluate the energy consumption of two prominent techniques, namely the PRISM encoder by Ramchandran [12][14] and the Wyner-Ziv encoder by Girod [15], [16]. Our experiments show that the PRISM encoder always consumes more energy (on average $64.93 \mathrm{~mJ} /$ frame) than the Wyner-Ziv encoder $(35.22 \mathrm{~mJ} /$ frame). Based on our evaluation, we identify the main energy consuming blocks in each of these

${ }^{1}$ Because intra encoder and decoder have almost the same complexity, therefore, intra coding scheme of predictive video coding paradigm is suitable when both the transmitter and the receiver are energy-constrained, whereas distributed video coding is suitable for base station based WSN deployments in which only the transmitter is considered to be energy-constrained. 
encoding schemes and propose modification to improve those blocks' energy efficiency. We show that significant energy savings can be realized for both DVC encoders by the proposed modifications.

Generally, the energy required to transmit one bit exceeds the energy required for a single local computation by a significant $\operatorname{margin}^{2}$ [17]. Therefore, all the video encoding schemes for WSNs use higher number of local computations to achieve the maximum possible compression and reduce the overall energy consumption. However, our results contradict this belief and report that energy depleted during video compression is significantly higher than the energy consumption during video transmission.

The rest of this paper is organized as follows: Section II describes the experimental setup. A brief background and energy consumptions of PVC and DVC video encoding alternatives are discussed in Sections III and IV, respectively. Conclusions are summarized in Section V.

\section{EXPERIMENTAL SETUP}

For our experiments, we employed $\mathrm{C} / \mathrm{C}++$ implementations of all video encoders used in this paper. For the PVC paradigm, we use the H.264/AVC reference implementation (JM 13.2) [7] for inter and intra encoding. Both inter and intra encoding were performed using the Main profile at 12 frames per second. For inter encoding, the order of the encoded frames was I-B-P-B-P-B-I. Default settings were used for the rest of the parameters. For the DVC paradigm, we implemented the PRISM and Wyner-Ziv encoders in $\mathrm{C} / \mathrm{C}++$ by closely following the description presented in [13] and [16] respectively. For judicious evaluation with PVC, we used $4 \mathrm{x} 4$ H.264 integer DCT for both of these encoders. For PRISM, the thresholds values $T_{1}$ and $T_{2}$ were set equal to 4.58 and 2046.5 respectively [14]. For Wyner-Ziv encoder, we used a $\mathrm{GOP}^{3}$ size of 2; i.e. odd numbered frames are encoded as key frames using H.264 Intra Main profile while the even numbered frames are Wyner-Ziv encoded using $\bar{M}^{4}$ quantization matrix defined in [16]. For the Motion JPEG2000, we used the Open JPEG Project implementation (OpenJPEG v1.3) [9].

Video sensor in our sensors network testbed comprise of a Stargate [3] gateway and a TelosB [4] mote. The $\mathrm{C} / \mathrm{C}++$ implementations for all video coding schemes are compiled via arm-linux-gcc/g++ 3.3.2 and run on the Stargate platform. Their compressed bit streams are transmitted via the attached TelosB mote. The computational energy depletion for Stargate platform was evaluated by calculating the CPU cycles consumed by each program and multiplying with the energy depletion per cycle count, given in TABLE I. The transmission energy depletion for TelosB mote was calculated similarly. Since the compressed data must be transmitted in 802.15.4 MAC data frames, the total number of transmitted bits includes the compressed data plus 9 bytes of header for every 93 bytes of actual frame payload [18]. The total energy

${ }^{2}$ For example, for our Stargate video sensor, the energy required for a single bit transmission is $4 \mu \mathrm{J}$ whereas the energy consumed during one CPU clock cycle is $2.644 \mathrm{~nJ}$.

For GOP $=N$, every $l^{s t}$ frame is encoded as key frame and the other $N-1$ as are encoded as WZ frames.
TABLE I

ENERGY DEPLETIONS FOR SENSOR PLATFORMS

\begin{tabular}{|c|c|c|}
\hline & 1 Cycle Count $(\mathbf{n J})$ & 1 bit Tx $(\boldsymbol{\mu J})$ \\
\hline Stargate & 2.644 & - \\
\hline TelosB & 1.215 & 4.000 \\
\hline
\end{tabular}

TX $=$ TRANSMISSION;

depleted for any encoding scheme is the sum of computational energy for encoding, bitstream transmission energy and computational energy of the Micro Controller Unit (MCU) of the attached TelosB mote for the duration of the transmission mode.

The experiments were performed for the first 96 frames of mobile, carphone, and foreman video sequences. Throughout the paper, all the energy consumption values are stated on per frame basis, unless otherwise stated. All of these videos sequences are in YUV format (decomposition 4:2:0), and of QCIF resolution (176 x 144). All these video sequences have different content characteristics. Specifically, the mobile sequence has a continuous relative motion of objects with some movement in the camera; the carphone sequence has a non-uniform changing background with more motion in the foreground [19]; and the foreman sequence has sharp motion and significant low complexity content. This content diversity enables us to comprehensively analyze the behavior and energy depletions of the present encoding schemes.

In the next two sections section, we present experimental results of the energy depletion of different encoding options in both the PVC and DVC paradigm. These two paradigms correspond to two different WSN communication scenarios: PVC is suitable for sensor- sensor communication, while DVC is designed for sensor-base-station communication. We present our performance evaluation keeping the following questions in mind: 1) What video encoding options (inter/intra/DVC video coding) provide better energy efficiency? 2) How much energy efficiency is provided by these different video encoding options? 3) What is the impact of different video characteristics (mobility, frequency content) on energy efficiency? 4) In what proportions do local computations for encoding and bits transmissions contribute to the overall energy depletion at the sensors? 5) Is it possible to propose some modifications to these existing encoders which could achieve further energy savings? These questions are intertwined and are addressed empirically in the next two sections.

\section{ENERGy EFFICIENCY Of PREDICTIVE VIDEO CODING}

In this section, we present a brief background and experimental results of the energy depletion of two encoding options (inter and intra video coding) of the Predictive Video Coding (PVC) paradigm. Next, we show how energy depletion of intra coding scheme can be reduced further.

\section{A. PVC Background}

Predictive Coding is the basis for most of the renowned video coding standards like MPEG-4 and H.264 [5]. PVC encoders use motion estimation to predict the next frame based on the previous few frames and encode the residual of the actual and the predicted frame. Two variants of this 
TABLE II

TOTAL ENERGY (COMPUTATIONS + TRANSMISSION) DEPLETIONS FOR ENCODING SCHEMES

\begin{tabular}{|c|c|c|c|c|c|c|c|c|c|c|c|}
\hline \multirow{3}{*}{ Sequence } & \multirow{3}{*}{$\begin{array}{c}\text { H.264 } \\
\text { Inter }\end{array}$} & \multirow{3}{*}{$\begin{array}{c}\text { H.264 } \\
\text { Intra }\end{array}$} & \multirow{3}{*}{$\begin{array}{c}\text { MJPEG2K } \\
\text { Intra }\end{array}$} & \multirow{3}{*}{ PRISM } & \multirow{3}{*}{ PRISM* } & \multicolumn{6}{|c|}{ Wyner-Ziv encoder } \\
\hline & & & & & & \multicolumn{3}{|c|}{ H.264 Key Frames } & \multicolumn{3}{|c|}{ MJPEG2K Key Frames } \\
\hline & & & & & & GOP $=2$ & GOP $=4$ & GOP $=8$ & GOP $=2$ & GOP $=4$ & GOP $=8$ \\
\hline Mobile & 769.86 & 64.64 & 20.39 & 62.67 & 48.52 & 37.85 & 25.15 & 18.28 & 12.42 & 11.74 & 12.15 \\
\hline Carphone & 757.88 & 57.71 & 11.42 & 68.41 & 50.53 & 33.56 & 21.82 & 16.11 & 9.16 & 9.43 & 10.31 \\
\hline Foreman & 763.32 & 57.74 & 13.65 & 63.71 & 46.90 & 34.26 & 22.49 & 17.48 & 9.69 & 10.24 & 11.39 \\
\hline
\end{tabular}

All Energy Values Are In Milli Joules;

paradigm which are used to balance the compressioncommunication tradeoff are: inter encoding and intra encoding [5], [6]. While inter coding significantly reduces the bit transmission energy depletion by exploiting motion compensation/estimation, it consumes a lot of computational energy to achieve this compression. Intra encoding, on the other hand, forgoes the motion compensation block and encodes each frame as an image. Since motion compensation/prediction is the most compute-intensive block in a video encoder, intra-coding scheme clearly saves a lot of computational energy but yields more bits for transmission. We will elaborate on these performance tradeoffs in next subsections.

\section{B. PVC Energy Consumption}

Let us first evaluate the energy consumed by the conventional inter and intra coding schemes of the PVC paradigm. The energy consumption of both these coding schemes on the Stargate video sensor is shown in TABLE II. Inter coding approximately consumes $769.86 \mathrm{~mJ} /$ frame of energy for mobile, $757.88 \mathrm{~mJ} /$ frame for carphone, and $763.32 \mathrm{~mJ} /$ frame for foreman. On the other hand, intra coding consumes approximately $64.64 \mathrm{~mJ} /$ frame of energy for mobile, $57.71 \mathrm{~mJ} /$ frame for carphone, and $57.74 \mathrm{~mJ} /$ frame for foreman. We can see that, for all of the three sequences, inter coding is significantly more expensive than intra coding because of the highly-complex motion compensation/estimation block.

It should also be noted that the energy depletions for the three video sequences are different; mobile consumes relatively high amount of energy as compared to carphone and foreman. This is because of continuous movement in the whole scene of the mobile sequence which increases the computations while encoding and also affects the accuracy of inter and/or intra prediction. Based on these results, it is quite evident that inter coding is not a suitable video coding alternative for low energy sensors. In other words, when both communication ends are complexity-constrained then intra coding should be used for video compression.

Figure 1 (a) and (b) show the percentages in which encoding computations and bit transmissions contribute to the overall energy depletion. In contrast to common belief, for both inter and intra coding, the computational energy depletion constitutes the major portion (on average $99.97 \%$ for inter and $98.48 \%$ for intra) of the total energy consumption as compared to the bit transmission energy (on average $0.03 \%$ for inter and $1.52 \%$ for intra), and is thus the dominating factor in determining the overall energy consumption in a video sensor network. a Computations $\square$ Transmissions $\quad$ Computations $\square$ Transmissions

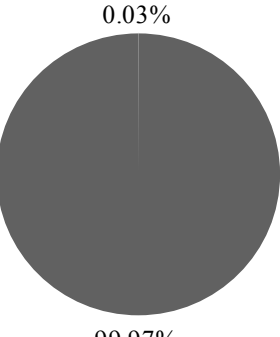

$99.97 \%$

(a) Inter encoding

Figure 1: Distribution of energies for predictive video coding.

\section{PVC Energy Consumption after Improvements}

At this point, we have established that intra coding is the better compression option for sensor-sensor video communication in WSNs. However, in the previous subsection, we presented the results of conventional intra coding based on the H.264/AVC encoder [5], [6]. We note that instead of performing intra coding using a video coder, compression can also be performed by a simple and optimized image coding algorithm. Using an image coding algorithm can decrease the complexity of video encoding, thereby reducing energy consumption on the motes. To this end, we now encode the video using the simple Motion JPEG2000 (MJPEG2K) encoder [8].

The energy consumptions of MJPEG2K based intra coding on the Stargate video sensor is shown in TABLE II. Figure 2 shows the comparative plot of the energy depletions for both H.264 and MJPEG2K based intra coding. We can see that, although H.264 intra coding achieves better compression than MJPEG2K intra coding (see Figure 3), MJPEG2K still saves a significant amount of energy; approximately $44.25 \mathrm{~mJ} /$ frame of energy for mobile, $46.29 \mathrm{~mJ} /$ frame for carphone, and $44.09 \mathrm{~mJ} /$ frame for foreman. H.264 intra coding contains intra prediction and de-blocking filter blocks which achieve a greater degree of compression but at the expense of a higher number of computations. MJPEG2K uses simple image coding techniques and therefore requires significantly lesser complexity and energy consumption.

From Figure 4, we can again observe that, on average, the computational energy depletion constitutes the major portion $(72.85 \%)$ of the total energy consumption and contribution of the bit transmission energy is still much less in comparison $(27.15 \%)$. Thus encoding computations are still the dominating factor in determining the overall energy consumption in a video sensor network. This is contrary to the commonly-held belief that higher compression (i.e., fewer bit 


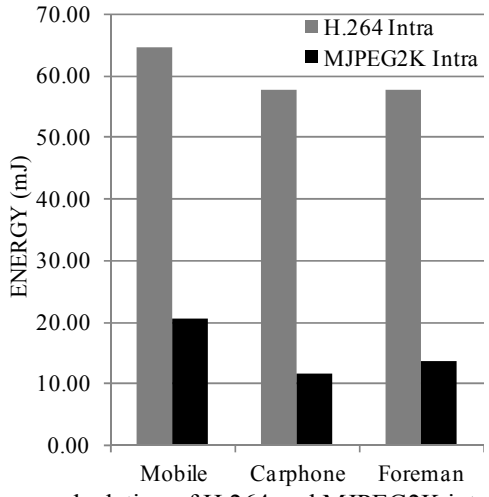

Figure 2: Energy depletion of H.264 and MJPEG2K intra encoding.

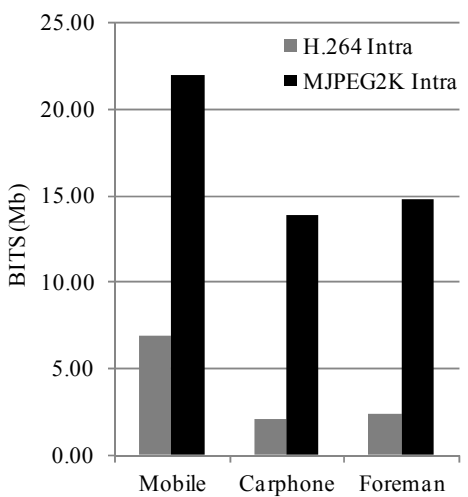

Figure 3: Compression efficiency of H.264 and MJPEG2K intra

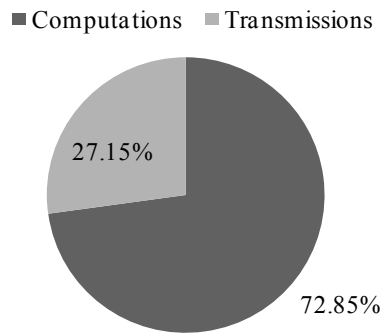

Figure 4: Distribution of energies for MJPEG2K intra coding.

transmissions) should be achieved at the cost of higher number of local computations.

\section{Energy EFFiciency Of Distributed Video CODING}

In this section, we present a brief background and experimental results of the energy depletion of two encoding options (PRISM and Wyner-Ziv video coding) of the Distributed Video Coding (DVC) paradigm and also show how the energy depletion for both the PRISM and Wyner-Ziv encoders can be reduced further.

\section{A. DVC Background}

DVC is a new video coding alternative that employs a lightweight encoder and a complex decoder. Two theoretical results on Distributed Source Coding by Slepian-Wolf [10] and Wyner-Ziv [11] state that, the same rate-distortion performance for joint encoding and decoding of two correlated sources can also be achieved by their separate encoding and joint decoding. Based on these information-theoretic results [10], [11], two practical DVC encoders were proposed in [12][16]; namely PRISM [12]-[14] and Wyner-Ziv encoders [15], [16]. Brief description of these codecs follows.
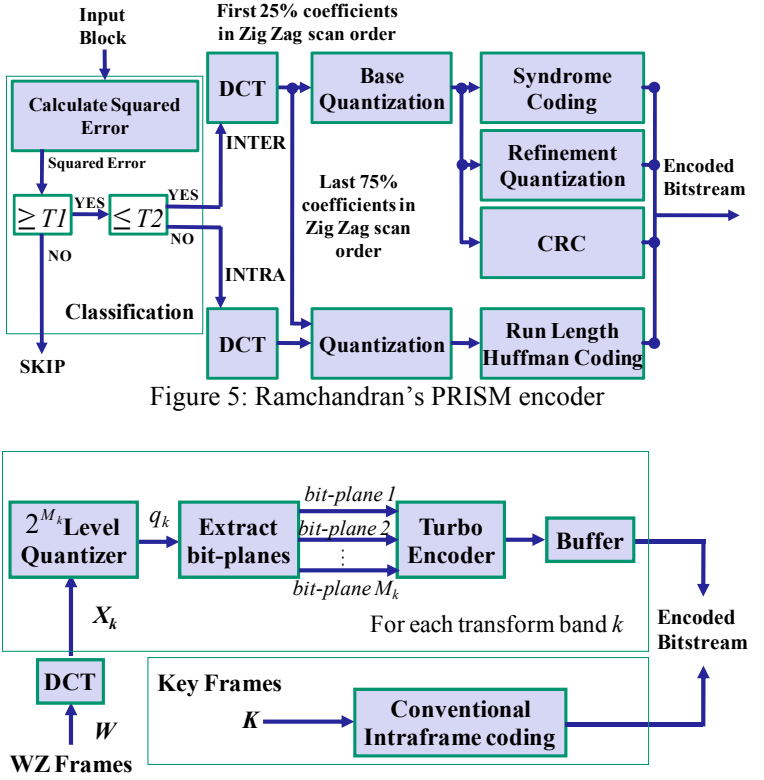

Figure 6: Girod's Wyner-Ziv encoder.

\section{1) PRISM Encoder}

The PRISM DVC encoder [12], [13] is shown in Figure 5. The PRISM encoder of [13] divides each frame into spatial blocks and encodes each block separately. For each block, at the pixels level it calculates the squared error between the current block and the co-located block in the previous frame. Based on the value of this square error, it classifies each block as SKIP, INTRA or INTER. If the squared error value of a block is less than the threshold $T 1$, this block is not encoded at all. If squared error value is greater than the threshold $T 2$, the block is intra encoded through DCT, Quantization and Runlength Huffman coding. The blocks having squared error value between these two thresholds (INTER blocks) are encoded by splitting some of its DCT coefficients on Base Quantization, Syndrome coding, CRC, and Refinement Quantization track and the rest on the INTRA coding track.

\section{2) Wyner-Ziv Encoder}

The Wyner-Ziv Video encoder [15], [16] shown in Figure 6 divides the frames of the sequence into two classes: Key frames and Wyner-Ziv (WZ) frames. Key frames are coded using conventional intra-encoding. The WZ frames are encoded using DCT, Quantization and Turbo coding. After the $4 \times 4$ DCT of all the blocks of each WZ frame, the corresponding transform coefficients of these blocks are grouped together to form 16 coefficient bands. The quantized symbols $q_{k}$ obtained from $2^{M_{k}}$ level uniform scalar quantization of each coefficient band $X_{k}$, are arranged to form $M_{k}$ bitplanes. All bit-planes are then encoded through the turbo encoder.

\section{B. DVC Energy Consumption}

We now compare the energy consumed by the PRISM and Wyner-Ziv coding schemes. The energy consumptions of both schemes on the Stargate video sensors are shown in TABLE II. Figure 7 shows the plots of the energy depletion of both PRISM and Wyner-Ziv coding. PRISM coding consumes, approximately, $62.67 \mathrm{~mJ} /$ frame of energy for mobile, 


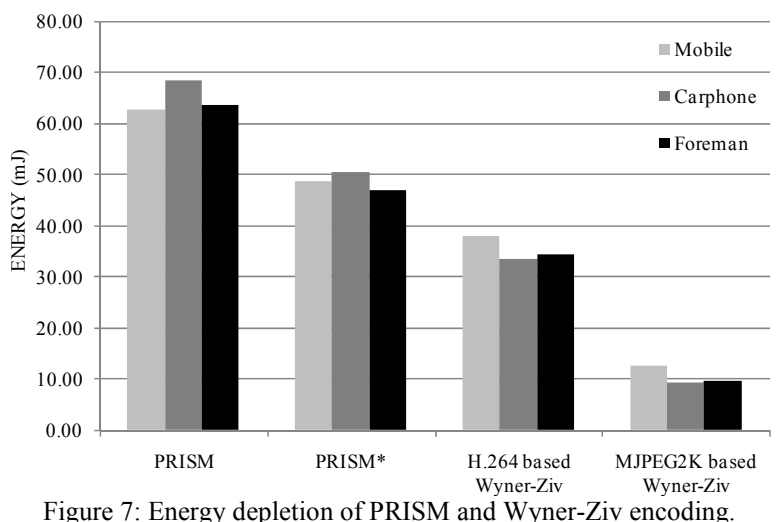

$68.41 \mathrm{~mJ} /$ frame for carphone, and $63.71 \mathrm{~mJ} /$ frame for foreman. On the other hand, Wyner-Ziv coding consumes, approximately, $37.85 \mathrm{~mJ} /$ frame of energy for mobile, $33.56 \mathrm{~mJ} /$ frame for carphone, and $34.26 \mathrm{~mJ} /$ frame for foreman. We can see that, for all of the three sequences, Wyner-Ziv coding has significantly better energy efficiency than PRISM coding. This is because of the classification and two-pass Runlength Huffman coding blocks in PRISM (see Figure 5). The classification block is computationally expensive because it is based on the calculation of squared error of all the pixels in each block. Similarly, the Huffman coding block, builds the Huffman tree in the first pass and then does compression in the second pass. On the other hand the Wyner-Ziv encoder operates on the DCT blocks of the whole WZ frame at the same time.

As expected, the Wyner-Ziv encoder depletes the most amount of energy for mobile and the least amount of energy for the carphone sequence. In Figure 8, we plotted the total energy depletions for the whole 96 frames in order to highlight the distribution of energy depletion among the Key and WZ frames of H.264 based Wyner-Ziv encoder with GOP $=2$. It is worth to note that, on average, for encoding the same number of frames, key frames consume 6 to 8 times more energy than that of WZ frames. In other words, the most complex block in Wyner-Ziv encoder is the intra coding of key frames.

Another interesting point that is worth mentioning here is that, unlike inter, intra or Wyner-Ziv coding, the energy depletion for the three video sequences is different for the PRISM encoder. The energy depletion decreases as both foreground and background movement in scenes of the sequences increases. Therefore, carphone (only movement in foreground) has the highest energy depletion and mobile (continuous movement in whole scene) has the least energy consumption. This is generally because in a sequence like carphone the classification block, based on the squared error value, marks most of the blocks in a frame as INTER blocks. Since the INTER blocks in PRISM are encoded by splitting their DCT coefficients on both encoding tracks, they contribute more towards the computational energy depletion. In contrast, for foreman and mobile comparatively less energy is depleted because less blocks are marked as INTER and more blocks are encoded through the DCT, Quantization and Huffman Coding track.

Figure 9 (a) and (c) show the proportion of energy depletion resulting from encoding computations and bit transmissions

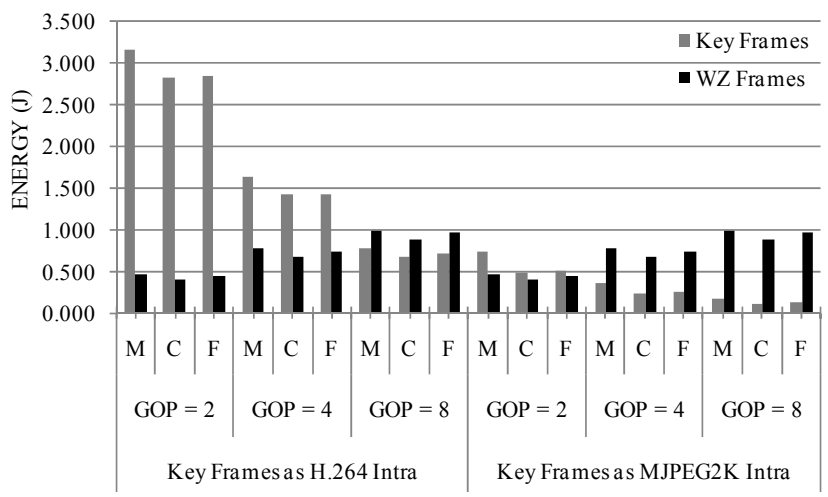

Figure 8: Energy depletion of H.264 and MJPEG2K based Wyner-Ziv coding on $\mathrm{GOP}=2,4,8$ for Mobile $(\mathrm{M})$, Carphone $(\mathrm{C})$ and Foreman $(\mathrm{F})$.

for PRISM and Wyner-Ziv encoders. As in the case of the PVC paradigm, the major portion of energy depletion results from encoding computations $(92.26 \%$ for PRISM and $97.46 \%$ for Wyner-Ziv); the contribution from bit transmissions is significantly smaller $(7.74 \%$ for PRISM and $2.54 \%$ for Wyner-Ziv). This implies that the same approach of reducing the encoding computations which worked for PVC will also be effective in reducing overall energy depletion for DVC. This approach is investigated further in the following subsection.

\section{DVC Energy Consumption after Improvements}

Although DVC encoders in general consume less amount of energy as compared to their complex decoders, both of these DVC approaches can be slightly modified to further reduce their energy consumptions.

As discussed previously the most complex blocks in PRISM encoder are: Calculation of Squared Error and Two-pass Runlength Huffman Coding. We can make PRISM more energy efficient by finding the low-complexity workarounds for these blocks. In our modification, labeled as PRISM*, we have calculated the squared error from just 4 pixels (taking 1 pixel from each block corner), and adjusted the thresholds accordingly. This reduces the complexity of squared error calculation by one fourth of the original encoder. It can be seen from Figure 7 that PRISM* saves, approximately, $14.15 \mathrm{~mJ} /$ frame of energy for mobile, $17.88 \mathrm{~mJ} /$ frame for carphone and $16.81 \mathrm{~mJ} /$ frame for foreman. More energy can be saved by employing a compression scheme which is more computationally efficient than Huffman coding.

Similarly, as discussed above, the most complex block in the Wyner-Ziv coding is the encoding of key frames through conventional intra coding. To save more energy, one option is to encode the key frames via MJEPG2K (as discussed in Section III.C). The second option is not to encode an equal number of key and WZ frames and increase the GOP size; i.e. encode less number of key frames as compared to WZ frames. This will result in more complexity for WZ block, more number of WZ bits transmissions and decoder complexity to effectively decode each WZ frame from a more distant key frame [15].

The energy consumptions of Wyner-Ziv encoder for GOP sizes of 2, 4 and 8 with their key frames encoded via H.264 intra and MJPEG2K are shown in TABLE II. Figure 8 plots the total energy consumptions of 96 frames, for all three GOP sizes via both of these key frame encoding options. For the 


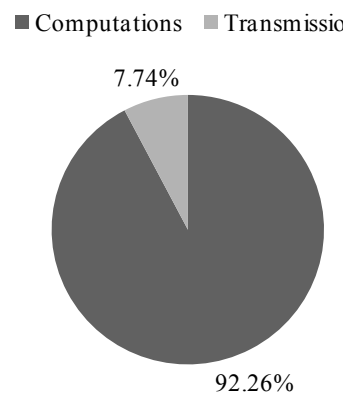

(a) PRISM
- Computations $\quad$ Transmissions

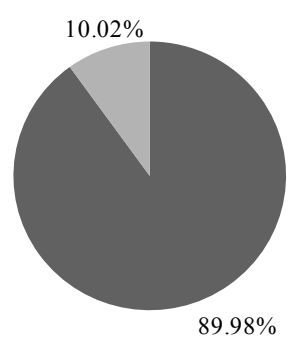

(b) PRISM*
- Computations $\square$ Transmissions

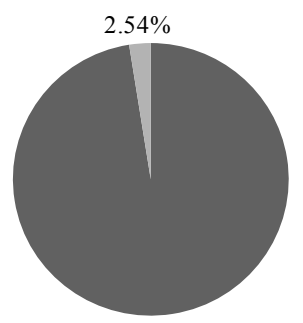

$97.46 \%$
- Computations Transmissions

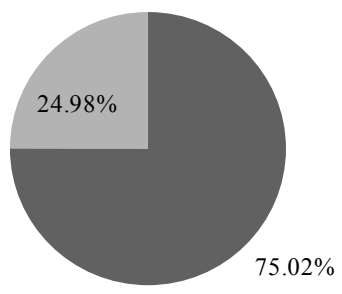

Figure 9: Distribution of energies for Original and Improved DVC encoder

case when key frames are encoded via H.264 intra, the energy consumption for all the three sequences decreases as the GOP size increases. The maximum energy is saved for the case of $\mathrm{GOP}=8 . \mathrm{MJPEG} 2 \mathrm{~K}$ encoding of key frames, in comparison, results in a significant reduction in overall energy consumption. For encoding the same number of frames (GOP =2), key frames and WZ frames consume almost the same amount of energy. Also note that, as the GOP size increases, the amount of energy depletion of WZ frames also increases. Thus for MJPEG2K based Wyner-Ziv encoder, mobile consumes the least energy for GOP $=4(11.74 \mathrm{~mJ} /$ frame $)$ while carphone and foreman consume the least energies for GOP $=2$ $(9.16 \mathrm{~mJ} /$ frame and foreman $9.69 \mathrm{~mJ} /$ frame respectively).

Figure 9 (b) and (d) show the energy depletion proportions for PRISM*, MJPEG2K based Wyner-Ziv encoders respectively $(\mathrm{GOP}=2)$. For PRISM*, encoding computations deplete the major portion $(89.98 \%)$ of the total energy; the bit transmissions deplete $10.02 \%$ of the total energy. Comparison of energy depletion values for PRISM and PRISM* verifies that overall energy depletion has been reduced by reducing the computational energy. As highlighted above, the MJPEG2K based Wyner-Ziv coding consumes almost the same energy for all GOP sizes. Therefore, in Figure 9 (d), we present the proportions of computational and transmission energy drains only for the case of GOP $=2$. We can observe that, approximately one fourth of the total energy is drained as part of bit transmissions and the rest is consumed as part of encoding computations. Based on the preceding discussion it is quite obvious that DVC encoders also tend to follow the same trend in terms of energy depletion i.e. most of the energy depletion results due to encoding computations, the contribution from bit transmissions is minor in comparison.

\section{CONCLUSION}

We compared different WSN video encoding schemes in Predictive and Distributed Video Coding paradigms on a Stargate based wireless video platform. For PVC paradigm, our results indicated that inter coding always consumes large amounts of energy, and therefore is not a suitable option for encoding video on low energy sensor motes. Furthermore, our results have shown significant energy savings can be achieved by utilizing Motion JPEG2000 based intra coding rather than H.264 based intra coding. For the DVC paradigm, our evaluation pointed out that the PRISM encoder depletes more energy than the Wyner-Ziv encoder. Furthermore, we proposed complexity reducing modifications to both DVC encoders which can introduce more energy savings in the
DVC encoders. Finally, for all the encoding schemes evaluated in this paper, we have established that, contrary to common belief, local computations are the major source of energy drain and not bit transmissions.

\section{REFERENCES}

[1] T.H.S. Krishnamurthy, et al. "An Energy-Efficient Surveillance System Using Wireless Sensor Networks," The Second International Conference on mobile Systems, Applications, and Services (MobiSys), June 2004.

[2] A.S. Tanenbaum, C. Gamage, and B. Crispo, "Taking Sensor Networks from the Lab to the Jungle," Computer: The magazine of the IEEE Computer Society, August, 2006.

[3] Crossbow Stargate Mote Specifications. <http://www.xbow.com>.

[4] Crossbow TelosB Mote Specifications. <http://www.xbow.com>.

[5] "Draft ITU-T Recommendation and Final Draft International Standard of Joint Video Specification," ITU-T Rec. H.264 and ISO/IEC 14496-10 AVC, May 2003.

[6] T. Wiegand, G. J. Sullivan, G. Bjøntegaard, and A. Luthra, "Overview of the H.264/AVC video coding standard," IEEE Transactions on Circuits and Systems for Video Technology, vol. 13, no. 7, pp. 560--576, July 2003.

[7] H.264/AVC Software Coordination<http://iphome.hhi.de/suehring/tml/>

[8] ISO/IEC 15444-3 Motion-JPEG2000 (JPEG2000 Part 3), 2002.

[9] The Open JPEG Project, U. Catholique de Louvain, Belgium, www.tele.ucl.ac.be/ PROJECTS/OPENJPEG.

[10] D. Slepian and J. K. Wolf, "Noiseless coding of correlated information sources," IEEE Transaction on Information Theory, vol. 19, pp. 471490, July 1973

[11] A. D. Wyner and J. Ziv, "The rate-distortion function for source coding with side information at the decoder," IEEE Trans. Inform. Theory, vol. IT-22, pp. 1-10, Jan. 1976.

[12] R. Puri and K. Ramchandran, "PRISM: A new robust video coding architecture based on distributed compression principles," Allerton Conference on Communication, Control, and Computing, Oct. 2002.

[13] R. Puri and K. Ramchandran, "PRISM: A video coding architecture based on distributed compression principles," Tech. Rep. No. UCB/ERL M03/6, ERL, UC Berkeley, March 2003.

[14] J. E. Fowler, "An Implementation of PRISM Using QccPack," Tech. Rep. MSSU-COE-ERC-05-01, Mississippi State ERC, Mississippi State University, January 2005.

[15] B. Girod, A. Aaron, S. Rane, D. Rebollo-Monedero, "Distributed Video Coding," Proceedings of the IEEE, vol .93, no. 1, pp. 71-83, January 2005.

[16] A. Aaron, S. Rane, E. Setton, and B. Girod, "Transform-domain WynerZiv codec for video," Proc. SPIE Visual Communications and Image Processing, San Jose, CA, Jan. 2004.

[17] T. T. Kwok, Y. Kwok, "Computation and Energy Efficient Image Processing in Wireless Sensor Networks Based on Reconfigurable Computing," International Conference on Parallel Processing Workshops (ICPPW'06), 2006.

[18] IEEE standard for part 15.4: Wireless MAC and PHY specifications for low rate WPAN. IEEE Std 802.15.4, IEEE, New York, NY, Oct. 2003.

[19] A. H. Sadka, Compressed Video Communications. John Wiley \& Sons, Ltd, 2002. 